\title{
A New Analytic Method of Multistate Single-component System Reliability Model
}

\author{
Xie Jingwei, Huang Peng and Liu Gang \\ Naval University of Engineering, Wuhan 430000 , China
}

\begin{abstract}
For the reliability modeling of multistate single-component system, single maintenance bench provides the preventive maintenance and alternative maintenance services on the basis of system performance level following the stochastic detection strategy. Phase-type distribution is employed in place of exponential distribution and other typical distributions to describe the stochastic time variable in the reliability modeling process in a unified manner. Through matrix analysis, the analytic expressions for reliability indicators including system steady-state availability, mean time between failures (MTBF) and failure rate of system are obtained. A numerical application is presented to verify the applicability of the model and demonstrate the influence of preventive maintenance threshold and preventive maintenance rate on system reliability.
\end{abstract}

\section{Introduction}

In the ship, nuclear power and aviation fields, large equipments feature complicated structural relationship, and their parts undertake all kinds of functions. As time passes by, the system often has different levels of performance. If the conventional binary state reliability theory is followed in the analysis and modeling of such system to roughly divide the system into two states, i.e. operation and failure, it is obviously difficult to satisfy the actual needs of reliability engineering. Hence, multistate system theory has become one of the research focuses in the reliability field ever since it was put forth in the 1980s [1].

Meanwhile, preventive maintenance is often conducted to guarantee the performance of equipment, so as to effectively reduce the considerable losses caused by the failure of large complicated equipment. Wang et al. [2] utilized the periodic preventive maintenance strategy to establish the reliability model for storage system and $k / n$ system. Chen [3] adopted different preventive maintenance strategies for different levels of parts, and put forward the age-based combined multistage classification and preventive maintenance model. It may be simple and easy to perform the periodic or scheduled preventive maintenance, but it is often difficult to carry out the timely maintenance, so maintenance resources will be wasted. Hence, the maintenance based on the state of system after detection appears to be more economical and reasonable. Yang [4] employed the age-based replacement and periodic detection strategy to study the optimal time between preventive maintenances, time between detections of competing failure system, as well as the number of detections.
In the above model, it is often considered that failure time and maintenance time are subject to exponential distribution, normal distribution, Weibull distribution and other typical distributions, which may make it less difficult to analyze the model, but limit the model to some special cases. To improve the applicability of model and maintain its good analytical ability under the typical distribution, Neuts [5] put forth the PH distribution in 1975 , as $\mathrm{PH}$ distribution could keep the easy calculation of exponential distribution, while favorably fitting other distributions on the non-negative axis. In this way, $\mathrm{PH}$ distribution could be applied extensively in the reliability field. Eryilmaz [6] assumed that the stochastic time variables in the model were subject to $\mathrm{PH}$ distribution, and studied the reliability of extreme impact model, continuous impact model and common impact model on this basis.

Above all, this paper assumes that a single-component system has a single maintenance bench, and employs the stochastic detection strategy to perform preventive maintenance based on the performance level of the system, and carry out the corrective maintenance during the complete failure. Meanwhile, considering the problems of strict constraints and limited applicability of the past reliability model, $\mathrm{PH}$ distribution is utilized to describe the hold time, time between detections, preventive maintenance time, and corrective maintenance time of the system at each performance level, build a more applicable reliability model, and obtain the expression for important reliability parameters including system steady-state availability and reliability functions, etc.

\section{Model Assumption}

It is assumed that a single-component system has multiple

Corresponding author: solehp@aliyun.com 
performance levels, and stochastic detection strategy is employed to perform preventive maintenance considering the performance level of system, and carry out corrective maintenance during the complete failure of system. The assumption is further extended as follows:

1) The internal performance level of system can be classified into $\{1, \cdots, m, m+1, \cdots, n, n+1\}$. Let $i$ stand for the current performance level of system, so the internal performance level of system can be classified into three types as follows: when $1 \leq i \leq m$, the internal performance of system is at the intact state; when $m+1 \leq i \leq n$, the internal performance of system is at the common state, i.e. the system can operate normally, but in poor performance; when $i=n+1$, the system is at the failure state.

2) The hold time of system at each performance level $i$ is subject to the continuous $\mathrm{PH}$ distribution, which has $f_{i}$ -order PH expression $\left(\boldsymbol{\alpha}_{i}, \boldsymbol{T}_{i}\right)$.

3) Let $p_{i y}(1 \leq i, y \leq n)$ stand for the probability that the internal performance level of system decreases from $i$ to $y$.

$$
\boldsymbol{P}=\left(\begin{array}{cc}
\boldsymbol{P}_{1} & \boldsymbol{P}_{12} \\
& \boldsymbol{P}_{2}
\end{array}\right), \quad \boldsymbol{P}^{0}=\left(\begin{array}{c}
\boldsymbol{P}_{1}^{0} \\
\boldsymbol{P}_{2}^{0}
\end{array}\right)
$$

In which, $\boldsymbol{P}_{1}$ and $\boldsymbol{P}_{2}$ denote the probability matrix that the internal performance of system experiences the internal transition between contact state and common state respectively. $\boldsymbol{P}_{12}$ is the probability matrix that the internal performance of system transmits from intact state to common state, while $\boldsymbol{P}_{1}^{0}$ and $\boldsymbol{P}_{2}^{0}$ stand for the probability matrix that the internal performance of system enters intact state or common state respectively.

4) The time between detections is subject to the discrete $\mathrm{PH}$ distribution, and has the $\varepsilon$-order $\mathrm{PH}$ expression $(\boldsymbol{\eta}, \boldsymbol{M})$;

(5) The preventive maintenance time is subject to the continuous $\mathrm{PH}$ distribution, and has the $z$-order $\mathrm{PH}$ expression $(\boldsymbol{\beta}, \boldsymbol{S})$;

6) The corrective maintenance time is subject to the continuous $\mathrm{PH}$ distribution, and has the $k$-order $\mathrm{PH}$ expression $(\boldsymbol{\theta}, \boldsymbol{U})$.

\section{Model Construction}

\subsection{System State Space}

It is assumed that the system performance is at the $x$ phase of the level $i$ at the time $t$; the detection phase is $h$; and the maintenance bench performs preventive maintenance and corrective maintenance at the phases $r_{1}$ and $r_{2}$ respectively. Considering the performance level of system and the operation mode of maintenance bench, the system state space can be represented by $\Omega$, $\Omega=\left\{H_{1} \cup H_{2} \cup H_{3}\right\}$.

When $H_{1}=\{i, x, h\}$, the system operates normally, its performance is at the intact state, and the detection phase is at $h$, in which $1 \leq i \leq n, 1 \leq x \leq f_{i}, 1 \leq h \leq \varepsilon$;

When $H_{2}=\left\{r_{1}\right\}$, the system is stopped, its performance is at the common state, and the maintenance bench performs the preventive maintenance of system, in which $1 \leq r_{1} \leq z$;

When $H_{3}=\left\{r_{2}\right\}$, the system fails, and the maintenance bench performs the corrective maintenance, in which $1 \leq r_{2} \leq k$.

\subsection{Infinitesimal Generator}

Considering the above division of system state spaces, the infinitesimal generator $\boldsymbol{Q}$ of the Markov chain is obtained as follows:

$$
\boldsymbol{Q}=\left(\begin{array}{lll}
\boldsymbol{Q}_{11} & \boldsymbol{Q}_{12} & \boldsymbol{Q}_{13} \\
\boldsymbol{Q}_{21} & \boldsymbol{Q}_{22} & \\
\boldsymbol{Q}_{31} & & \boldsymbol{Q}_{33}
\end{array}\right)
$$

If the internal performance level of system is detected, it will be introduced through matrixes $\boldsymbol{U}_{1}$ and $\boldsymbol{U}_{2}$, and let $o_{1}=\sum_{i=1}^{m} f_{i}, o_{2}=\sum_{i=m+1}^{n} f_{i}, o=o_{1}+o_{2}=\sum_{i=1}^{n} f_{i}$.

$$
\boldsymbol{U}_{1}=\left(\begin{array}{cc}
\boldsymbol{I}_{o_{1}} & \boldsymbol{0} \\
\boldsymbol{0} & \boldsymbol{0}
\end{array}\right)_{o \times o}, \quad \boldsymbol{U}_{2}=\left(\begin{array}{cc}
\boldsymbol{0} & \boldsymbol{0} \\
\boldsymbol{0} & \boldsymbol{I}_{o_{2}}
\end{array}\right)_{o \times o}
$$

$\boldsymbol{Q}_{11}$ stands for the internal transition of system in the state space $H_{1}$.

$$
\begin{aligned}
\boldsymbol{Q}_{11}= & \left\{\operatorname{blkdiag}\left(\boldsymbol{T}_{1}, \cdots, \boldsymbol{T}_{m}\right)+\operatorname{blkdiag}\left(\boldsymbol{T}_{1}, \cdots, \boldsymbol{T}_{m}\right) \boldsymbol{P} .\right. \\
& \left.\operatorname{blkdiag}\left(\boldsymbol{\alpha}_{1}, \cdots, \boldsymbol{\alpha}_{m}\right)\right\} \otimes \boldsymbol{I}_{\varepsilon}+\boldsymbol{V}_{1} \otimes \boldsymbol{M}^{0} \boldsymbol{\eta}+\boldsymbol{I}_{o} \otimes \boldsymbol{M}
\end{aligned}
$$

In which, the first term is the transition of the internal performance of system at the level $i(1 \leq i \leq n)$; the second term means that it is detected that the internal performance level of system is at the intact state; the third term implies no detection in the system, so the phase of the internal performance level of system does not change. Similarly, the followings can be obtained:

$$
\begin{aligned}
& \boldsymbol{Q}_{22}=\boldsymbol{S} ; \quad \boldsymbol{Q}_{33}=\boldsymbol{U} ; \quad \boldsymbol{Q}_{12}=\boldsymbol{V}_{1} \boldsymbol{e}_{o} \otimes \boldsymbol{M}^{0} \boldsymbol{\beta} ; \\
& \boldsymbol{Q}_{13}=\operatorname{blkdiag}\left(\boldsymbol{T}_{1}^{0}, \cdots, \boldsymbol{T}_{m}^{0}\right) \boldsymbol{P}^{0} \otimes \boldsymbol{e}_{\varepsilon} \boldsymbol{\theta} ; \\
& \boldsymbol{Q}_{21}=\left[\boldsymbol{S}^{0} \otimes \boldsymbol{\alpha}_{1} \otimes \boldsymbol{\eta} 0\right] ; \\
& \boldsymbol{Q}_{31}=\left[\boldsymbol{U}^{0} \otimes \boldsymbol{\alpha}_{1} \otimes \boldsymbol{\eta} 0\right] ;
\end{aligned}
$$

\subsection{Steady-state Probability Vector}

When the system enters the steady state, the probabilities of states in the infinitesimal generator form a steady-state probability vector $\pi=\left(\pi_{1}, \pi_{2}, \pi_{3}\right)$, which satisfies the following equation:

$$
\left\{\begin{array}{l}
\pi Q=0 \\
\pi e=1
\end{array}\right.
$$

Equation (7) can be written into:

$$
\pi_{1} Q_{11}+\pi_{2} Q_{21}+\pi_{3} Q_{31}=0
$$




$$
\begin{aligned}
& \pi_{1} Q_{12}+\pi_{2} Q_{22}=0 \\
& \pi_{1} Q_{13}+\pi_{3} Q_{33}=0
\end{aligned}
$$

After solving the above equations, the steady-state probability vector $\pi=\left(\pi_{1}, \pi_{2}, \pi_{3}\right)$ can be obtained for each state space.

\section{System Reliability Indicators}

\subsection{System Steady-state Availability}

System steady-state availability refers to the probability when a system is at the normal state after long-term operation. For the model constructed in this paper, when the system operates normally, the system remains at the state of operation, so the steady-state availability of system $A_{1}$ is obtained as follows:

$$
A_{1}=\boldsymbol{\pi}_{1} \boldsymbol{e}
$$

When the system operates normally and stops for preventive maintenance, i.e. the system remains at the state of operation and the state of preventive maintenance, the system does not enter the state of shutdown due to failure, so the steady-state availability of system $A_{2}$ is obtained as follows:

$$
A_{2}=\pi_{1} \boldsymbol{e}+\boldsymbol{\pi}_{2} \boldsymbol{e}
$$

\subsection{System Reliability Function}

System reliability function is the probability that the system does not fail before the time $t$. For the model in this paper, system reliability refers to the probability that the system is at the state of operation and the state of preventive maintenance or not at the state of failure before the time $t$. The failure state in the system $H_{3}$ is combined into absorptive state, so the corresponding state transition matrix $Q^{*}$ is as follows:

$$
\boldsymbol{Q}^{*}=\left(\begin{array}{ll}
\boldsymbol{Q}_{11} & \boldsymbol{Q}_{12} \\
\boldsymbol{Q}_{21} & \boldsymbol{Q}_{22}
\end{array}\right)
$$

State transition matrix $Q^{*}$ represents the transition between the states when the system is not at the state of failure. Let $\boldsymbol{W}_{i}(t)$ stand for the probability that the system is at the state $H_{i}(i=1,2)$, $\boldsymbol{W}(t)=\left(\boldsymbol{W}_{1}(t), \boldsymbol{W}_{2}(t)\right)$. Moreover, $\boldsymbol{W}_{i}(t)$ and $\boldsymbol{Q}^{*}$ satisfy the following differential equation:

$$
\left\{\begin{array}{l}
\boldsymbol{W}^{\prime}(t)=\boldsymbol{W}(t) \boldsymbol{Q}^{*} \\
\boldsymbol{Q}^{*}(0)=\boldsymbol{\varphi}_{1}
\end{array}\right.
$$

The differential equation is solved to obtain the system reliability function $R(t)$.

$$
R(t)=\boldsymbol{\varphi}_{1} \exp \left(\boldsymbol{Q}^{*} t\right) \boldsymbol{e}
$$

In which, $\varphi_{1}=\left(\boldsymbol{\alpha}_{1} \otimes \boldsymbol{\gamma} \otimes \boldsymbol{\eta}, \boldsymbol{0}\right)$.

\subsection{Mean Time between Failures (MTBF) of System}

The MTBF of system refers to the time taken for the system to exit and then reenter the failure state. In this model, the time between failures contains the time of operation and the time of preventive maintenance. When the system exits the failure state $H_{3}$, it operates normally. When the system reenters the state $H_{3}$, it fails. According to the definition of Phase-type (PH), the MTBF of system is subject to $\mathrm{PH}$ distribution, and has the $o \varepsilon+z$-order irreducible $\left(\boldsymbol{\varphi}_{1}, \boldsymbol{Q}^{*}\right)$ expression. Hence, the MTBF of system is obtained as follows:

$$
M T B F=-\varphi_{1} Q^{*-1} e
$$

\subsection{Mean Time between Maintenances (MTBM) of System}

The MTBM of system is the time taken for the system to exit and then reenter the maintenance state. Hence, the maintenance states of system $\mathrm{H}_{2}$ and $\mathrm{H}_{3}$ can be combined into absorptive state, so the corresponding state transition matrix $\boldsymbol{O}$ is as follows:

$$
\boldsymbol{O}=\boldsymbol{Q}_{00}
$$

According to the definition of Phase-type (PH), the MTBM of system is subject to $\mathrm{PH}$ distribution, and has the $o \varepsilon$-order irreducible $\left(\boldsymbol{\varphi}_{2}, \boldsymbol{O}\right)$ expression, in which $\boldsymbol{\varphi}_{2}=\left(\boldsymbol{\alpha}_{1} \otimes \boldsymbol{\gamma} \otimes \boldsymbol{\eta}, \boldsymbol{0}\right)$. According to the definition, the MTBM of system can be obtained as follows:

$$
M T B M=-\boldsymbol{\varphi}_{2} \boldsymbol{O}^{-1} \boldsymbol{e}
$$

\subsection{Failure Rate of System}

Failure rate of system is the probability that the system enters the state of maintenance due to failure, and it is denoted by $r$. Considering the system state transition matrix $\boldsymbol{Q}$, there is:

$$
r=\pi_{1} Q_{13} e
$$

\section{Numerical Application}

\subsection{Applicability Verification of the Model}

It is know that an equipment system has 5 performance levels in which $\{1,2,3,4\}$ mean that the system is at the state of operation, and $\{5\}$ indicates the failure state of system. The hold time of system at each performance level is as given in Table 1 .

Table 1. Hold time distribution of system at each performance level

\begin{tabular}{|c|c|}
\hline$i$ & Distribution of hold time \\
\hline \multirow{2}{*}{1} & $\boldsymbol{\alpha}_{1}=(1)$ \\
& $\boldsymbol{T}_{1}=(-0.500)$ \\
\hline 2 & $\boldsymbol{\alpha}_{2}=(1,0,0,0)$ \\
\hline
\end{tabular}




\begin{tabular}{|c|c}
\hline \multirow{3}{*}{3} & $\boldsymbol{T}_{2}=\left(\begin{array}{cccc}-1.660 & 0.500 & 0 & 0 \\
0 & -1.583 & 0.468 & 0 \\
0 & 0 & -1.620 & 0.483 \\
0 & 0 & 0 & -1.589\end{array}\right)$ \\
\hline 4 & $\boldsymbol{T}_{3}=\left(\begin{array}{ccc}-2.000 & 0.500 & 0.10 \\
0.250 & -1.000 & 0.150 \\
0.500 & 0 & -1.500\end{array}\right)$ \\
\hline $\boldsymbol{T}_{4}=\left(\begin{array}{cccc}-3.320 & 0 & 3.320 & 0 \\
0.010 & -3.350 & 0 & 3.310 \\
0 & 0 & -3.318 & 0 \\
3.346 & 0 & 0.002 & -3.348\end{array}\right)$
\end{tabular}

The probability matrix of transition between system performance levels:

$$
\boldsymbol{P}=\left(\begin{array}{cccc}
0 & 0.880 & 0.100 & 0.010 \\
0 & 0 & 0.875 & 0.110 \\
0 & 0 & 0 & 0.957 \\
0 & 0 & 0 & 0
\end{array}\right)
$$

Distribution of time between detections $\boldsymbol{\eta}=(1,0,0)$, $\boldsymbol{M}=\left(\begin{array}{ccc}-0.9 & 0.83 & 0 \\ 0 & -0.7 & 0.54 \\ 0 & 0 & -0.95\end{array}\right) ;$ distribution of time between preventive maintenances $\boldsymbol{b}=(1), \quad \boldsymbol{S}=(-1.2)$; distribution of time between corrective maintenances $\boldsymbol{\theta}=(1,0), \quad \boldsymbol{U}=\left(\begin{array}{cc}-2.5 & 2.5 \\ 2.43 & -2.5\end{array}\right)$

When $m=1$, i.e. the performance level $\{1\}$ is at the intact state, and $\{2,3,4\}$ is at the common state, data are submitted into the model to obtain the steady-state availability $A_{1}=0.8236, A_{2}=0.9985$; mean time between failures of system $M T B F=11.9588$; mean time between maintenances $M T B M=4.7768$; and failure rate of system $r=0.0035$. The system reliability function is as shown in Fig. 2.

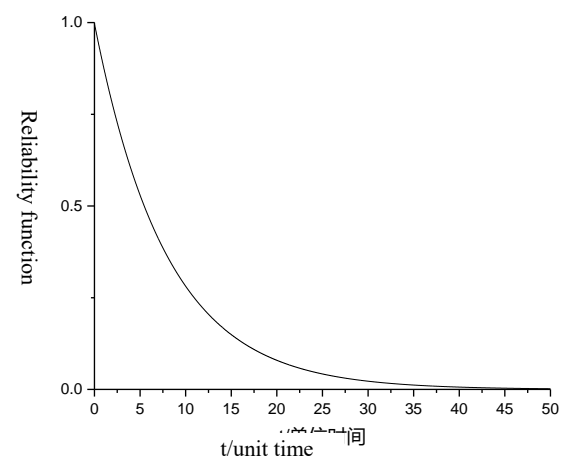

Fig 1. Relationship between system reliability and time

As shown in the calculation results, the application of
PH distribution in describing the stochastic time variables in the modeling process of reliability can effectively help obtain all reliability parameters, so the applicability of the model is improved considerably.

\subsection{Influence of Preventive Maintenance Threshold on System Reliability Indicators}

Let $m=1,2,3$, and substitute data into the model to obtain the results of reliability indicators as presented in Table 2.

Table 2. Influence of preventive maintenance threshold $m$ on reliability indicators

\begin{tabular}{|c|c|c|c|}
\hline$m$ & 1 & 2 & 3 \\
\hline$A_{1}$ & 0.8236 & 0.8472 & 0.8653 \\
\hline$A_{2}$ & 0.9985 & 0.9843 & 0.9526 \\
\hline$M T B F$ & 11.9588 & 12.0356 & 13.7751 \\
\hline$M T B M$ & 4.7768 & 6.5562 & 9.7589 \\
\hline$r$ & 0.0035 & 0.0078 & 0.0137 \\
\hline
\end{tabular}

Increasing the preventive maintenance threshold can enhance the probability of system at the state of operation, time between failures and time between preventive maintenances, but the failure rate of system also increases correspondingly.

\subsection{Influence of Preventive Maintenance Rate on System Reliability Indicators}

To vividly display the influence of preventive maintenance rate and preventive maintenance threshold on system reliability, exponential distribution is utilized as a special case of $\mathrm{PH}$ distribution to present the influence of preventive maintenance rate on system reliability. Assuming that time between preventive maintenances is subject to the exponential distribution of the parameter $\lambda$, there is PH distribution, $\boldsymbol{\beta}=1, \boldsymbol{S}=\lambda$, so the results are as shown in Fig. 3-Fig. 7.

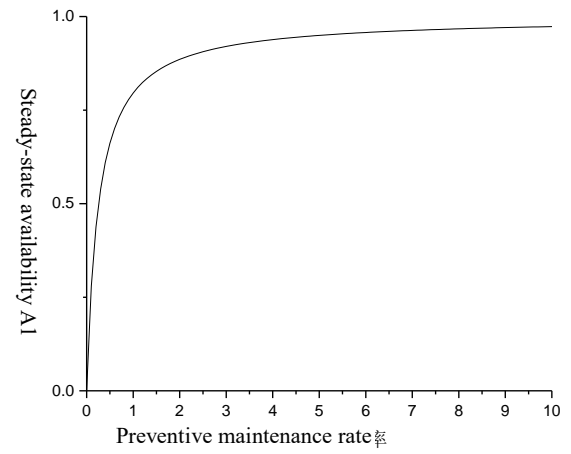

Fig 2. Influence of preventive maintenance rate on steady-state availability 


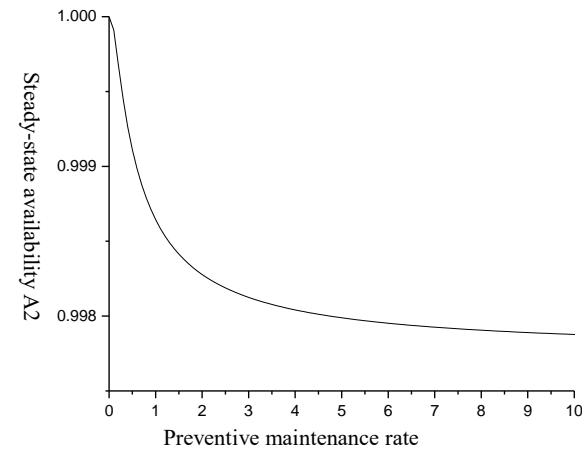

Fig 3. Time between failures with influence of preventive maintenance rate on steady-state availability

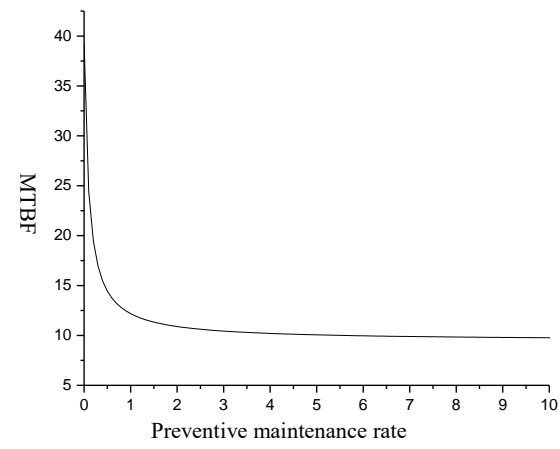

Fig 4. Influence of preventive maintenance rate on MTBF

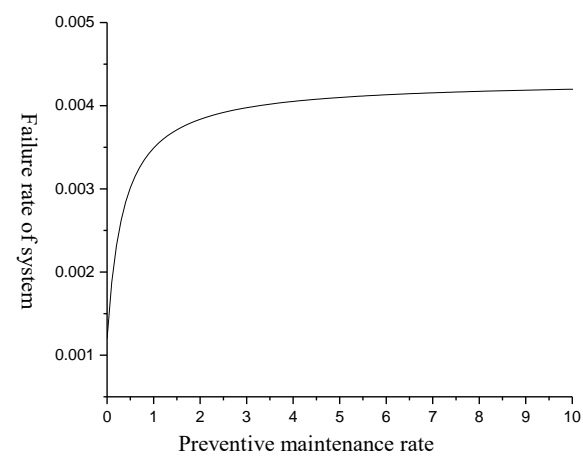

Fig 5. Influence of preventive maintenance rate on failure rate of system

As shown in Fig. 2-Fig. 5, the probability of system at the state of operation increases gradually along with the increase of preventive maintenance rate, so steady-state availability $A_{1}$ and failure rate of system increase as well, and become stable gradually. The time between preventive maintenances decreases relatively, causing the decrease of steady-state availability $A_{2}$ and MTBF, which become stable gradually. However, MTBM is only related to the preventive maintenance threshold, and unrelated to the preventive maintenance rate.

According to the above analysis of the results, system reliability is affected to some extent when preventive maintenance threshold or preventive maintenance rate is changed. To achieve the requirements for reliability in the practical engineering, preventive maintenance threshold and preventive maintenance rate should be set in a reasonable manner.

\section{Conclusion}

This paper utilizes the stochastic detection strategy and PH distribution to describe the stochastic time variables of model in a unified manner, builds a multistate singlecomponent system reliability model, and obtains the expressions for reliability indicators including system steady-state availability, MTBF, MTBM, and failure rate of system. Additionally, a numerical application is presented to verify the applicability of the model, and demonstrate the influence of preventive maintenance threshold and preventive maintenance rate on system reliability indicators. In this way, this paper provides the theoretical support for selecting the preventive maintenance threshold and distributing the maintenance resources in a reasonable manner.

\section{References}

1. R. M. Barton and W. W. Damon: Reliability in a Multi-state System. C. 1974

2. B. Y. Wang, X. F. Wang and Z. H. Bie: Reliability Model of MMC Considering Periodic Preventive Maintenance. IEEE Transactions on Power Delivery. J. 32, 1535-1544(2017)

3. Z. Chen, T. B. Xia and E. S. Pan: Optimal multi-level classification and preventive maintenance policy for highly reliable products. International Journal of Production Research. J. 55, 2232-2250(2017)

4. L. Yang, X. B. Ma and R. Peng: A preventive maintenance policy based on dependent two-stage deterioration and external shocks. Reliability Engineering and System Safety. J. 160, 201-211 (2017)

5. M. F. Neuts and K.S. Meier: On the use of phase type distributions in reliability modelling of systems with two components. OR Spectrum. J. 2, 227-234(1981)

6. S. Eryilmaz: Computing optimal replacement time and mean residual life in reliability shock models. Computers and Industrial Engineering J. 103, 4045(2017) 